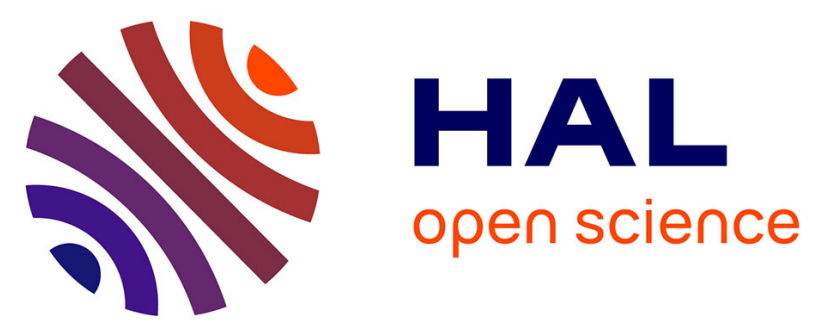

\title{
Immediate vs Delayed Stenting in ST-Elevation Myocardial Infarction: Rationale and Design of the International PRIMACY Bayesian Randomized Controlled Trial
}

E. Marc Jolicoeur, Nandini Dendukuri, Patrick Belisle, Grégoire Range, Geraud Souteyrand, Frédéric Bouisset, Gilles Zemour, Nicolas Delarche, Brahim Harbaoui, Erick Schampaert, et al.

\section{- To cite this version:}

E. Marc Jolicoeur, Nandini Dendukuri, Patrick Belisle, Grégoire Range, Geraud Souteyrand, et al.. Immediate vs Delayed Stenting in ST-Elevation Myocardial Infarction: Rationale and Design of the International PRIMACY Bayesian Randomized Controlled Trial. Canadian Journal of Cardiology, 2020, 10.1016/j.cjca.2020.01.019 . hal-02917540

\author{
HAL Id: hal-02917540 \\ https://hal.science/hal-02917540
}

Submitted on 29 Oct 2020

HAL is a multi-disciplinary open access archive for the deposit and dissemination of scientific research documents, whether they are published or not. The documents may come from teaching and research institutions in France or abroad, or from public or private research centers.
L'archive ouverte pluridisciplinaire $\mathbf{H A L}$, est destinée au dépôt et à la diffusion de documents scientifiques de niveau recherche, publiés ou non, émanant des établissements d'enseignement et de recherche français ou étrangers, des laboratoires publics ou privés. 


\title{
Immediate vs Delayed Stenting in ST-Elevation Myocardial Infarction: Rationale and Design of the International PRIMACY Bayesian Randomized Controlled Trial
}

\author{
E. Marc Jolicoeur, MD, MSc, MHS, ${ }^{a}$ Nandini Dendukuri, PhD, ${ }^{b}$ Patrick Belisle, MSc, ${ }^{c}$
} Grégoire Range, MD, ${ }^{\mathrm{d}}$ Geraud Souteyrand, $\mathrm{MD},{ }^{\mathrm{e}}$ Frédéric Bouisset, MD, ${ }^{\mathrm{f}}$ Gilles Zemour, MD, ${ }^{\mathrm{g}}$ Nicolas Delarche, MD, ${ }^{\mathrm{h}}$ Brahim Harbaoui, MD, ${ }^{\mathrm{i}}$ Erick Schampaert, MD, ${ }^{\mathrm{j}}$ Simon Kouz, MD, ${ }^{\mathrm{k}}$ Guillaume Cayla, MD, PhD, ${ }^{1}$ François Roubille, MD, PhD, ${ }^{\mathrm{m}}$ Ziad Boueri, MD, ${ }^{\mathrm{n}}$

Samer Mansour, MD, ${ }^{\circ}$ Xavier Marcaggi, MD, ${ }^{\mathrm{p}}$ Jean-Claude Tardif, MD, ${ }^{\mathrm{a}}$ Michael McGillion, RN, PhD, ${ }^{\mathrm{q}}$ Jean-François Tanguay, MD, ${ }^{\mathrm{a}} \mathrm{James}$ Brophy, MD, PhD, ${ }^{\mathrm{r}}$ Cheol Woong Yu, MD, PhD, ${ }^{s}$ Colin Berry, MBCHB, PHD, ${ }^{t}$ David Carrick, MBCHB, PHD, ${ }^{u}$ Dan Eik Høfsten, MD, ${ }^{\mathrm{v}}$ Thomas Engstrøm, MD, ${ }^{\mathrm{v}}$ Lars Kober, MD, ${ }^{\mathrm{v}}$ Henning Kelbæk, MD, ${ }^{\mathrm{w}}$ and Loic Belle, MD

\begin{abstract}
${ }^{a}$ Montreal Heart Institute, Université de Montréal, Montréal, Québec, Canada; ${ }^{b}$ Centre for Outcomes Research, McGill University Health Centre-Research Institute, Montreal, Quebec, Canada; ${ }^{C}$ Montreal Health Innovation Coordination Center, Montreal, Quebec, Canada; ${ }^{d}$ Department of Cardiology, Les Hopitaux de Chartres, EuretLoir, France; ${ }^{e}$ CHU-Clermont Ferrand, Clermont-Ferrand, France; ${ }^{f}$ Toulouse Rangueil University Hospital (CHU), Toulouse, France; ${ }^{g}$ Centre Hospitalier Pierre Nouveau Cannes, Cannes, France; ${ }^{h}$ Centre Hospitalier de Pau, Pau, France; ${ }^{i}$ Hôpital Croix-Rousse and Hôpital Lyon Sud, Hospices Civils de Lyon, Lyon, France; ${ }^{j}$ Hopital SacreCoeur, Interventional Cardiology, Université de Montréal, Montreal, Quebec, Canada; ${ }^{k}$ Centre Hospitalier Régional de Lanaudiere, Joliette, Quebec, Canada; ${ }^{l}$ Centre Hospitalier Universitaire Nimes, Université de Montpellier, Nimes, France; ${ }^{m}$ PhyMedExp, Université de Montpellier, INSERM, CNRS, Cardiology Department, CHU de Montpellier, Montpellier, France; ${ }^{n}$ Centre Hospitalier de Bastia, Bastia, France; ${ }^{\circ}$ Centre Hospitalier Universitaire de l'Université de Montréal, Université de Montréal, Montreal, Quebec, Canada; ${ }^{p}$ Department of Cardiology, Centre Hospitalier de Vichy, Vichy, France; ${ }^{q}$ Population Health Research Institute, McMaster University, Hamilton, Ontario, Canada; ' McGill University Health Centre, Division of Cardiology, Montreal, Quebec, Canada; ${ }^{\prime}$ Korea University Anam Hospital, Cardiovascular Center (Interventional Cardiology), Seoul, Republic of Korea; ${ }^{t}$ BHF Glasgow Cardiovascular Research Center, Institute of Cardiovascular and Medical Sciences, University of Glasgow, Glasgow; and West of Scotland Heart and Lung Center, Golden Jubilee National Hospital, Dunbartonshire, United Kingdom; "University Hospital Hairmyres, East Kilbride, Glasgow, United Kingdom; "Rigshospitalet-Copenhagen University Hospital, Department of Cardiology, Copenhagen, Denmark; ${ }^{w}$ Dept of Cardiology, Zealand University Hospital, Roskilde, Denmark; ${ }^{x}$ Hospital of Annecy, Centre Hospitalier Annecy Genevois, Annecy, France
\end{abstract}

\section{ABSTRACT}

Background: Primary percutaneous coronary intervention is used to restore blood flow in the infarct-related coronary artery, followed by immediate stenting to prevent reocclusion. Stents implanted in thrombus-laden arteries cause distal embolization, which paradoxically impairs myocardial reperfusion and ventricular function. Whether a strategy of delayed stenting improves outcomes in patients with acute ST-elevation myocardial infarction (STEMI) is uncertain.

Acute ST-elevation myocardial infarction (STEMI) is a leading cause of mortality and morbidity in Canada and globally. Despite optimal medical therapy and optimal reperfusion,

Corresponding author: Dr E. Marc Jolicoeur, Montreal Heart Institute, 5000 Bélanger Est, Montréal, Québec H1T 1C8, Canada. Tel.: +1-514-3763330; fax: +1-514-593-2155.

E-mail: marc.jolicoeur@icm-mhi.org

\section{RÉSUMÉ}

Contexte : L'intervention coronarienne percutanée (ICP) primaire permet de rétablir la circulation dans l'artère coronaire responsable de l'infarctus, et est immédiatement suivie de la pose d'une endoprothèse pour prévenir une nouvelle occlusion. Lorsqu'elle est implantée dans une artère remplie de caillots, l'endoprothèse provoque une embolie distale, ce qui peut paradoxalement nuire à la reperfusion du myocarde et à la fonction ventriculaire. On ne sait pas si reporter la pose de

STEMI continues to adversely affect outcomes, including reinfarction and heart failure. The consequences of STEMI impose significant personal, societal, and financial burdens. ${ }^{2}$ The recent improvements in prognosis post STEMI have capped, because cardioprotection strategies have not translated into clinical benefits. ${ }^{3}$

Compared with balloon angioplasty as a primary reperfusion therapy, stent implantation during primary percutaneous intervention (PCI) prevents early reocclusion. Although the 
Methods: The Primary Reperfusion Secondary Stenting (PRIMACY) is a Bayesian prospective, randomized, open-label, blinded end point trial in which delayed vs immediate stenting in patients with STEMI were compared for prevention of cardiovascular death, nonfatal myocardial infarction, heart failure, or unplanned target vessel revascularization at 9 months. All participants were immediately reperfused, but those assigned to the delayed arm underwent stenting after an interval of 24 to $\mathbf{4 8}$ hours. This interval was bridged with antithrombin therapy to reduce thrombus burden. In the principal Bayesian hierarchical random effects analysis, data from exchangeable trials will be combined into a study prior and updated with PRIMACY into a posterior probability of efficacy.

Results: A total of $\mathbf{3 0 5}$ participants were randomized across 15 centres in France and Canada between April 2014 and September 2017. At baseline, the median age of participants was 59 years, $81 \%$ were male, and $3 \%$ had a history of percutaneous coronary intervention. Results from PRIMACY will be updated from the patient-level data of 1568 participants enrolled in the Deferred Stent Trial in STEMI (DEFER; United Kingdom), Minimalist Immediate Mechanical Intervention (MIMI; France), Danish Trial in Acute Myocardial Infarction-3 (DANAMI3; Denmark), and Impact of Immediate Stent Implantation Versus Deferred Stent Implantation on Infarct Size and Microvascular Perfusion in Patients With ST Segment-Elevation Myocardial Infarction (INNOVATION, South Korea) trials.

Conclusions: We expect to clarify whether delayed stenting can safely reduce the occurrence of adverse cardiovascular end points compared with immediate stenting in patients with STEMI.

mortality benefit of stenting during primary PCI is modest compared with balloon angioplasty alone, ${ }^{4}$ prevention of late target vessel revascularization is well established. ${ }^{5}$ However, stents implanted to thrombus-laden arteries might paradoxically compromise coronary flow by eliciting distal emboli, and impair myocardial reperfusion, all of which can further induce myocardial damage and predispose to heart failure.

With evidence-based use of dual antiplatelet therapy, glycoprotein (Gp) IIb/IIIa blockade, and anticoagulation, the rates of subacute coronary reocclusion have decreased dramatically in patients with STEMI. ${ }^{6}$ This has led several groups around the world to reconsider the need for immediate stenting during primary PCI. ${ }^{7}$ They hypothesized that the potential drawbacks of immediate stenting in thrombus-laden arteries could be avoided by delaying stent implementation until after bridging adjunctive antithrombin therapy, allowing for a reduction in thrombus burden (Fig. 1). ${ }^{8}$

\section{The minimalist immediate mechanical intervention} approach in delayed stenting

Early meta-analyses of nonrandomized studies associated delayed stenting with fewer distal embolization and l'endoprothèse améliore les résultats chez les patients présentant un infarctus du myocarde avec élévation du segment ST (STEMI) aigu. Méthodologie : L'étude PRIMACY (Primary Reperfusion Secondary Stenting) est un essai bayésien prospectif mené en mode ouvert, avec répartition aléatoire et critère à l'insu visant à comparer l'efficacité de l'implantation d'une endoprothèse reportée à celle d'une implantation immédiate pour ce qui est de prévenir le décès d'origine cardiovasculaire, l'infarctus du myocarde non mortel, l'insuffisance cardiaque et la revascularisation d'un vaisseau cible non prévue à 9 mois chez des patients ayant subi un STEMI. Tous les participants ont été reperfusés sans délai, mais chez ceux qui ont été affectés au groupe d'implantation reportée, l'endoprothèse a été mise en place après un intervalle de 24 à $48 \mathrm{~h}$. Durant cet intervalle, les patients ont reçu de l'antithrombine afin de réduire la charge thrombotique. Aux fins de l'analyse principale des effets aléatoires au moyen d'un modèle hiérarchique bayésien, les données d'essais échangeables seront regroupées en une distribution de probabilité antérieure et mises à jour au moyen des données de l'étude PRIMACY pour obtenir une probabilité postérieure d'efficacité.

Résultats : Au total, 305 participants traités dans 15 centres situés en France et au Canada entre avril 2014 et septembre 2017 ont été répartis aléatoirement. Au départ, l'âge médian des participants était de 59 ans; $81 \%$ d'entre eux étaient des hommes, et $3 \%$ avaient déjà subi une ICP. Les résultats de l'étude PRIMACY seront mis à jour à partir des données recueillies auprès des 1568 participants admis aux études DEFER (Deferred Stent Trial in STEMI; Royaume-Uni), MIMI (Minimalist Immediate Mechanical Intervention; France), DANAMI-3 (Danish Trial in Acute Myocardial Infarction-3; Danemark) et Impact of Immediate Stent Implantation Versus Deferred Stent Implantation on Infarct Size and Microvascular Perfusion in Patients With ST SegmentElevation Myocardial Infarction INNOVATION, Corée du Sud).

Conclusions : Nous prévoyons ainsi préciser les données afin de déterminer si reporter l'implantation d'une endoprothèse permet de réduire sans danger le risque d'issue cardiovasculaire défavorable comparativement à l'implantation immédiate d'une endoprothèse chez les patients ayant subi un STEMI.

major adverse cardiovascular events. ${ }^{9}$ Delayed stenting is achieved through the minimalist immediate mechanical intervention approach, ${ }^{10}$ which stipulates that: (1) reperfusion of the infarct-related artery should be attempted with the tools least likely to injure the artery or cause distal emboli; and (2) upon reperfusion, the stent should be implanted only after the thrombus has significantly regressed using an adjuvant antithrombotic regimen. This approach assumes that clot lysis by the in situ endothelial and systemic fibrinolytic system combined with 24 hours or more of dual antiplatelet therapy combined with an intravenous antithrombin agent with or without the addition of GpIIb/IIIa blockade will reduce the thrombus burden and minimize the chances of no-reflow after stenting (Fig. 2). ${ }^{11}$

\section{Evidence for delayed stenting in patients with STEMI}

To date, 4 randomized clinical trials have tested the hypothesis of delayed stenting in patients with STEMI (Supplemental Table S1). In the Deferred Stent Trial in STEMI (DEFER), ${ }^{12}$ Minimalist Immediate Mechanical Intervention (MIMI), ${ }^{13}$ and Impact of Immediate Stent Implantation Versus Deferred Stent Implantation on Infarct Size 


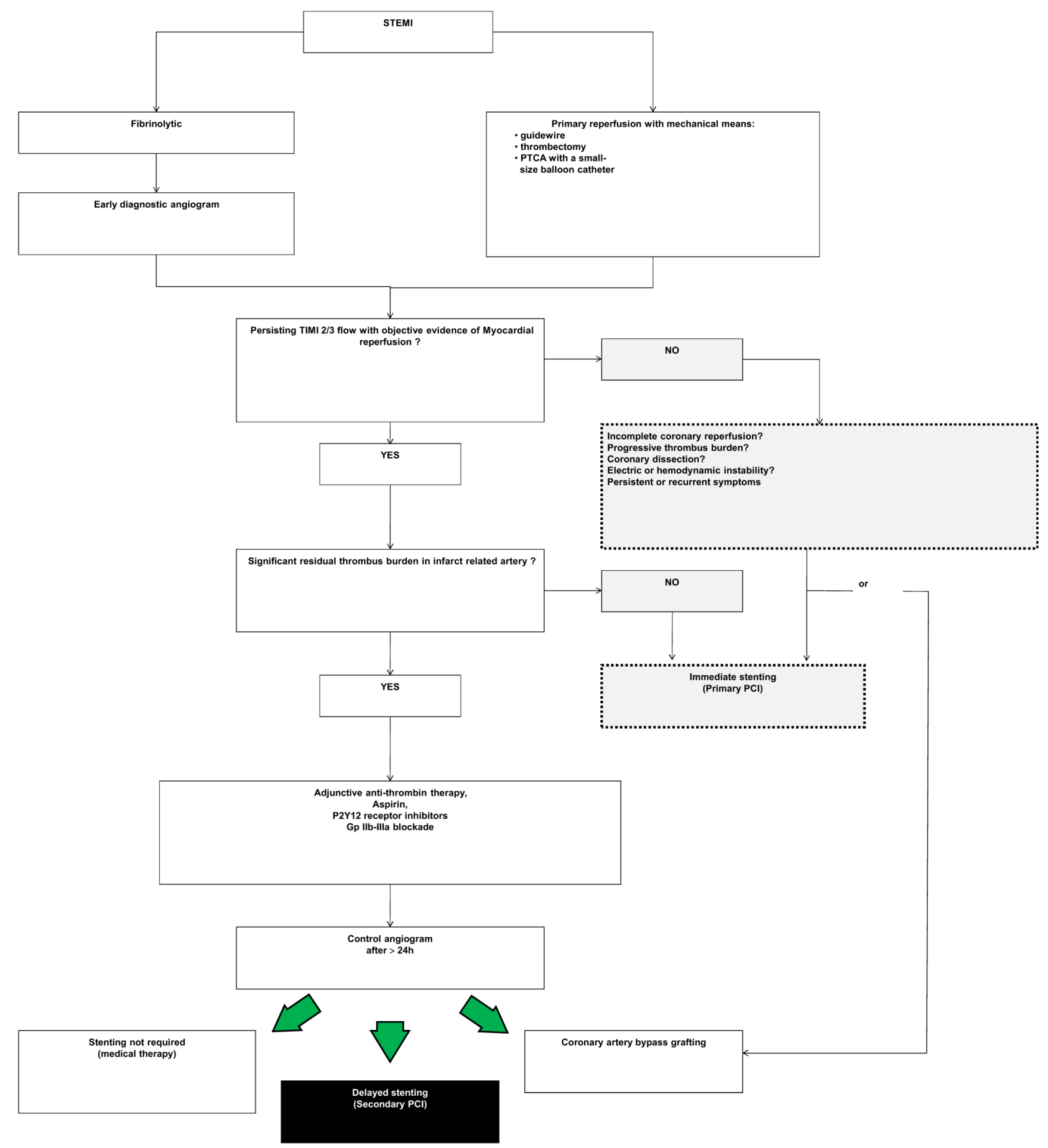

Figure 1. The concept of delayed stenting in patients with acute myocardial infarction. Gp, glycoprotein; PCI, percutaneous coronary intervention; PTCA, percutanous transluminal coronary angioplasty; STEMI, ST-elevation myocardial infarction; TIMI, Thrombolysis in Myocardial Infarction. Modified from Jolicoeur et al. ${ }^{8}$ with permission from Elsevier Inc.

and Microvascular Perfusion in Patients With ST SegmentElevation Myocardial Infarction (INNOVATION) ${ }^{94}$ trials the surrogate end point of myocardial tissue perfusion was investigated, whereas in the Third DANish Study of Optimal Acute Treatment of Patients With ST-elevation Myocardial Infarction (DANAMI-3-DEFER) cardiovascular outcomes were investigated. In the Danish Trial in Acute Myocardial Infarction-3 (DANAMI-3), delayed stenting did not meet the primary end point of all-cause death, heart failure, nonfatal infarction, or any unplanned target vessel revascularization (hazard ratio, 0.83; 95\% confidence interval, 0.56-1.20). ${ }^{15}$

\section{Bayesian designs in the study of medical strategies}

Bayesian inference is an alternative to the traditional "frequentist" Neyman-Pearson statistics. ${ }^{16}$ The Bayes theorem states that the posterior probability of an event is proportional to the product of the prior probability of the event and the 
measured likelihood, which provides the information in the current data set. Applied to clinical trials, Bayesian inference incorporates prior probabilities in testing a hypothesis and calculates the updated posterior probability for the hypothesis in question, after evidence from the ongoing trial is included. Bayesian analysis therefore allows for sequential learning as evidence accumulates; the degree of conviction in favour of a new treatment changes over the course of the trial.

\section{The PRIMACY Bayesian Trial}

\section{Design and objectives}

The Primary Reperfusion Secondary Stenting (PRIMACY) is a prospective, randomized, open-label, blinded end point, multicentre controlled trial in which the efficacy and the safety of delayed stent implantation in acute myocardial infarction are being tested (ClinicalTrials.gov NCT01542385; Fig. 3). The primary end point is the first occurrence of a composite end point consisting of the following events during a 9-month period: cardiovascular death, nonfatal myocardial infarction, heart failure, or unplanned target vessel revascularization. The primary safety end point is the occurrence of major bleeding over a 9-month period.

\section{Study population}

PRIMACY was conducted in 15 sites in Canada and France. Entry criteria are presented in Table 1. Patients who presented with a STEMI ( $<12$ hours of symptom onset) and an occluded infarct-related artery (Thrombolysis in Myocardial Infarction [TIMI] 0-1 flow grade), but successfully reperfused (TIMI 2-3 flow) with a minimalist immediate mechanical intervention technique were deemed eligible if their infarct-related artery remained patent for more than 10 minutes, with no signs of imminent reocclusion (degradation in TIMI flow, dissection) or deterioration in their clinical status. The protocol was approved by institutional review boards in all participating centres and written informed consent was obtained from all participants. The national funding agency or industry partners played no role in the conduct and oversight of the study.

\section{Randomization and concealment}

Participants were allocated in a 1:1 ratio to either arm using a centrally controlled computer-generated permuted block randomization stratified according to site. The study personnel randomized the participants during the primary PCI. The allocation sequence remained concealed in sealed envelopes until the study arm was assigned. Time zero was

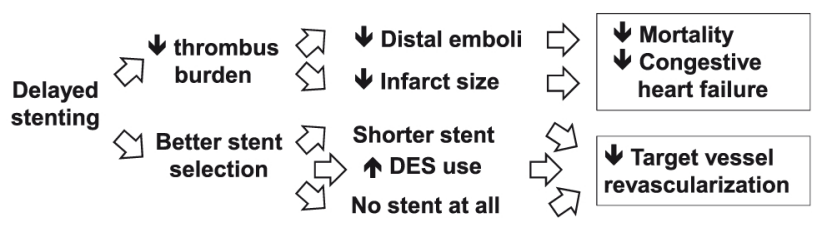

Figure 2. How delayed stenting might improve outcomes. DES, drugeluting stent. defined as the time of randomization. Because of the urgent and invasive nature of STEMI treatment, blinding was not possible. To minimize this bias, the members of the pre- and post probability board, the statisticians performing the analysis, the members of the Clinical Event Committee (CEC), and the members of the Data Safety Monitoring Board (DSMB) remained blinded to treatment assignment until after database lock.

\section{Study procedures and follow-up}

Participants were treated with aspirin $(250-650 \mathrm{mg})$ and ticagrelor $(180 \mathrm{mg})$ before their arrival in the catheterization laboratory. Occluded (TIMI flow 0-1) coronary arteries had to be reopened spontaneously after wire passage with or without thrombectomy aspiration catheter (operator decision). In cases when thrombectomy alone failed to reestablish flow, inflation of a small balloon catheter $(\leq 2.0 \mathrm{~mm})$ could be attempted. The choice of the anticoagulant during the reperfusion was left to the discretion of the operator, preferentially with unfractionated heparin bolus $(70-100 \mathrm{U} / \mathrm{kg})$ to maintain an activated clotting time above $\geq 225$ seconds, or with bivalirudin (initial bolus $0.75 \mathrm{mg} / \mathrm{kg}$, drip $1.75 \mathrm{mg} / \mathrm{kg} /$ h). Transradial artery access was strongly encouraged. In participants assigned to immediate stenting, all intravenous anticoagulation was stopped at procedure end, unless medically indicated. The use of GpIIb/IIIa inhibitors was left to the operator discretion in participants assigned to immediate stenting, but for those assigned to delayed stenting, GpIIb/ IIIa inhibitors had to be started (or continued) for 12-18 hours, followed by systemic anticoagulation with either unfractionated heparin, low molecular weight heparin, or bivalirudin until the delayed stent implantation. The choice between bare metal vs drug-eluting stent (Synergy everolimuseluting platinum chromium stent; Boston Scientific, Marlborough, MA) was left to the discretion of the operator, but the use of drug-eluting stent was strongly encouraged. Participants were assessed for adverse events at hospital discharge, and postprocedurally at 30 days (telephone), 3 months (telephone), and 9 months (in person visit; Table 2).

\section{Definitions of outcomes and adjudication of end points}

The definitions for cardiovascular death, nonfatal myocardial infarction, and heart failure were adapted from the previously validated criteria reported in the 2014 and 2017 American College of Cardiology/American Heart Association definitions for cardiovascular end point events in clinical tri$\mathrm{als},{ }^{11}$ and the third universal definition of myocardial infarction $^{17}$ (Table 3 and Supplemental Appendix S1). The definition for unplanned revascularisation of the target vessel was designed to capture the unique hazard related to delayed stenting, including any postrandomization event that would occur in the interval between reperfusion and delayed stent implantation, or any in-stent restenosis and or stent thrombosis that would occur post implantation. Unplanned revascularization of the target vessel therefore included any repeat PCI or coronary artery bypass grafting procedure to the infarct-related vessel that was prompted by unstable ischemic symptoms, and associated with either: (1) an acute deterioration of coronary flow caused by thrombus progression or dissection and resulting in recurrent symptoms or 


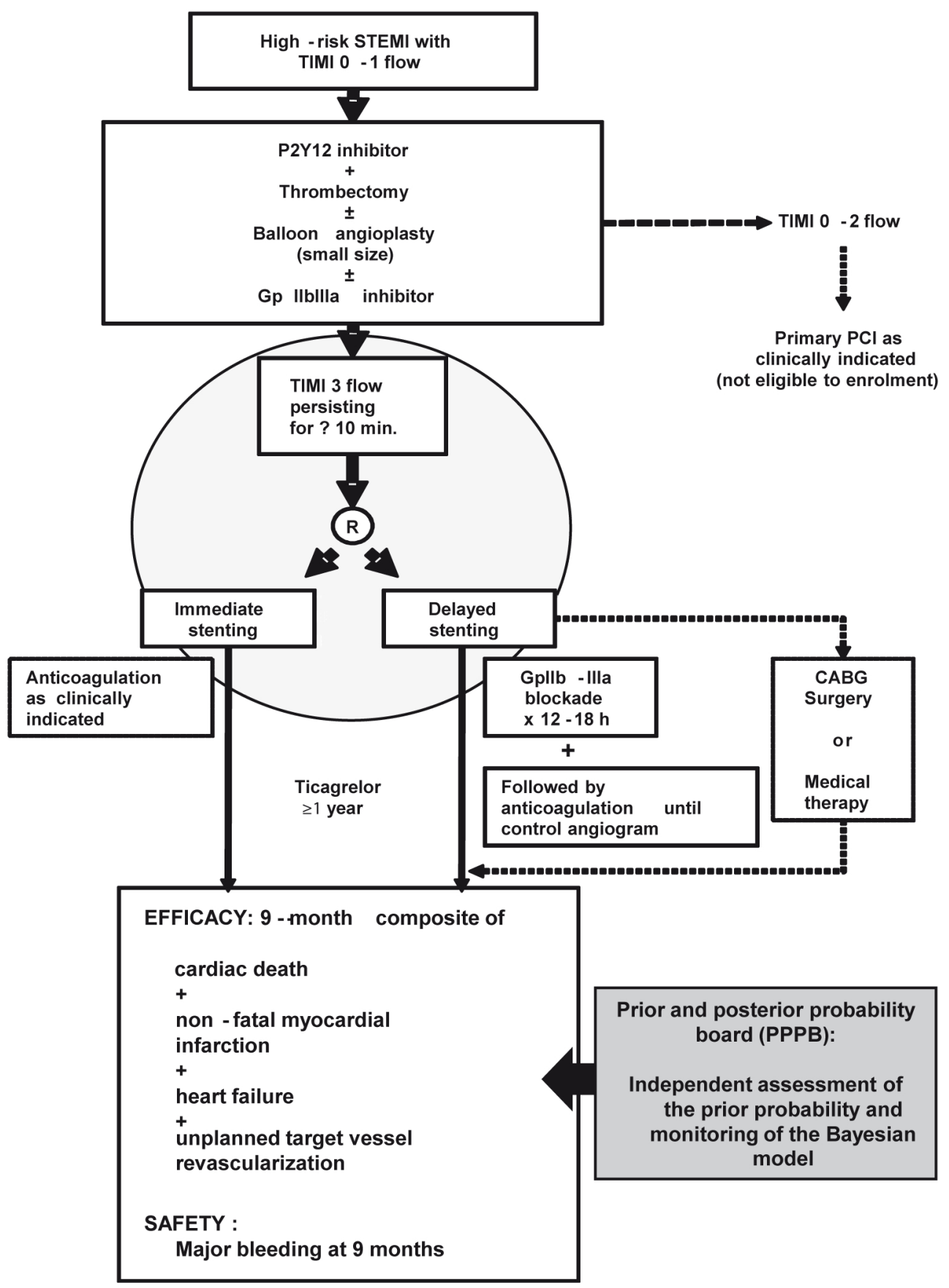

Figure 3. The Primary Reperfusion Secondary Stenting (PRIMACY) trial flow. CABG, coronary artery bypass grafting; Gp, glycoprotein; PCI, percutaneous coronary intervention; R, randomization; STEMI, ST-elevation myocardial infarction; TIMI, Thrombolysis in Myocardial Infarction.

electrocardiographic changes; or (2) a positive functional ischemia study and an angiographic minimal lumen diameter stenosis of $\geq 50 \%$ using quantitative coronary angiography; or (3) an angiographic minimal lumen diameter stenosis of at least $70 \%$ using quantitative coronary angiography in the absence of a positive functional ischemia study.

For the primary safety end point, major bleeding was defined using the universal Bleeding Academic Research Consortium criteria. ${ }^{18}$ All triggered events were adjudicated by an independent CEC blinded to assigned treatment using source documents in line with the CEC charter.

\section{Sample size calculation}

Bayesian sample size estimates were calculated using a method adapted from Joseph et al. in 2007. ${ }^{19}$ We used a customized $\mathrm{R}$ program ( $\mathrm{R}$ Foundation for Statistical Computing, Vienna, Austria), which took into account beta priors as inputs and simulated possible trial results on the basis of these priors. The final sample size required was that which reached a chosen accuracy in estimating between treatment differences on average over 10,000 simulations of the trial. In particular, given a set of design priors (which provide a range 
Inclusion criteria

Subjects must meet all of the following inclusion criteria to be eligible for participation in this study:

1. Patient age between 18 and 80 years

2. Signed informed consent

3. ST-segment elevation myocardial infarction, presenting within 12 hours of symptoms onset, and persisting for more than 20 minutes

4. ECG that fulfils any of the following criteria: $\geq 2$-mm ST elevation in 2 anterior or lateral leads; or $\geq 1$-mm ST elevation in 2 inferior leads; or new LBBB with at least $1-\mathrm{mm}$ concordant ST elevation

5. Infarct-related artery with a TIMI flow grade $0-1$ at baseline angiogram

6. Successful reperfusion (TIMI 2-3 flow), either spontaneously or after wire passage, thrombectomy, small size ( $\leq 2.0 \mathrm{~mm})$ angioplasty catheter, and persisting for more than 10 minutes

7. Infarct-related artery with a diameter $>2.5 \mathrm{~mm}$

Exclusion criteria

Subjects who meet any of the following exclusion criteria are not eligible to be enrolled in this study:

1. Previous STEMI in the qualifying coronary artery

2. Coronary dissection after reperfusion

3. STEMI caused by acute stent thrombosis or a venous or arterial bypass graft occlusion

4. Known significant left main disease, determined using angiography $(\geq 50 \%)$ or other imaging technologies, at time of screening

5. Cardiac condition requiring emergent or urgent surgical repair

6. Failed thrombolysis and rescue PCI

7. High risk of bleeding

8. Contraindication to either ticagrelor or GpIIb/IIIa inhibitors

9. STEMI with Killip III-IV or cardiogenic shock or presenting as sudden death, ventricular fibrillation, or sustained ventricular tachycardia

10. Women who are pregnant or breastfeeding

11. Known renal impairment (creatinine clearance $<20 \mathrm{~mL} / \mathrm{min}$ ) at time of screening

12. Other contraindication to PCI

13. Participation in another investigational drug or investigational device study within 30 days before randomization (participation to registries is allowed)

14. Any condition that in the opinion of the investigator would preclude compliance with the study protocol

ECG, electrocardiogram; Gp, glycoprotein; LBBB, left bundle branch block; PCI, percutaneous coronary intervention; STEMI, ST-elevation myocardial infarction; TIMI, Thrombolysis in Myocardial Infarction.

of expected rates in the treatment and control groups), we simulated trial results using our design and assuming a flat analysis prior (because no additional trials were reported when we planned the trial). The sources of design prior are as follows: for the control group, we used a beta $(48.4,153$.) prior and for the treatment group, we used a beta $(35.0,208.5)$ prior. These priors corresponded to a $95 \%$ prior range of primary end points between $18 \%$ and $30 \%$ vs $10 \%$ and $19 \%$ for the control and treatment group, respectively (Supplemental Fig. S1). The beta priors used had means and medians near the centre of their intervals. In particular, a beta (48.4, 153.0) prior has a mean of 0.24 , and a beta (35.0, $208.5)$ prior has a mean of 0.145 . These ranges were sourced from expert opinion, previous experience, and importantly from the anticipated rates of events cited in the literature at the start of the trial in 2014. In STEMI trials, the minimal clinically important absolute difference has historically been set at $1 \%$ for mortality, ${ }^{6}$ and $3 \%-4 \%$ for short-term rate of congestive heart failure. ${ }^{20}$ Delayed stenting is expected to yield an absolute reduction of $7.7 \%$ for the primary end points at 9 months, including reductions of approximately $3 \%$ for heart failure, $3.3 \%$ for revascularization, and $1 \%$ for mortality. In using a mean quantile for the posterior credible interval width set at $\pm 10 \%$, we estimated that a sample size of 304 participants would yield a 50\% chance of being adequate to obtain an accuracy of \pm 0.1 in estimating the effect between immediate and delayed stenting. The sample size was adjusted for expected dropouts and loss to follow-up (3\%) using the Lachin formula.

\section{Statistical analysis and integration of previous evidence}

PRIMACY will be the first to be analyzed as a stand-alone trial and will thereafter be updated with external evidence.

Table 2. Study procedures

\begin{tabular}{|c|c|c|c|c|c|c|}
\hline \multirow[b]{2}{*}{ Assessment } & \multicolumn{3}{|c|}{ In-hospital } & \multicolumn{3}{|c|}{ Follow-up } \\
\hline & Day 0 & Day $1-3$ & Discharge & Day 30 (phone) & 3 Months (phone) & 9 Months (in-person visit) \\
\hline Consent & $\sqrt{ }$ & $\sqrt{ }$ & & & & \\
\hline Baseline assessment & $\sqrt{ }$ & & & & & \\
\hline Physical exam & $\sqrt{ }$ & & $\sqrt{ }$ & & & $\sqrt{ }$ \\
\hline Electrocardiogram & $\sqrt{ }$ & & $\sqrt{ }$ & & & $\sqrt{ }$ \\
\hline Blood tests & $\sqrt{ }$ & & $\sqrt{ }$ & & & \\
\hline Biomarkers & $\sqrt{ }$ & $\sqrt{ }$ & & & & \\
\hline Left ventricular ejection fraction & & $\sqrt{ }$ & & & & \\
\hline NYHA functional status & & & $\sqrt{ }$ & $\sqrt{ }$ & $\sqrt{ }$ & \\
\hline Health-related quality of life & & & $\sqrt{ }$ & & & $\sqrt{ }$ \\
\hline Medications & $\sqrt{ }$ & $\sqrt{ }$ & $\sqrt{ }$ & $\sqrt{ }$ & $\sqrt{ }$ & $\sqrt{ }$ \\
\hline Serious adverse events & $\sqrt{ }$ & $\sqrt{ }$ & $\sqrt{ }$ & $\sqrt{ }$ & $\sqrt{ }$ & $\sqrt{ }$ \\
\hline
\end{tabular}

NYHA, New York Heart Association. 


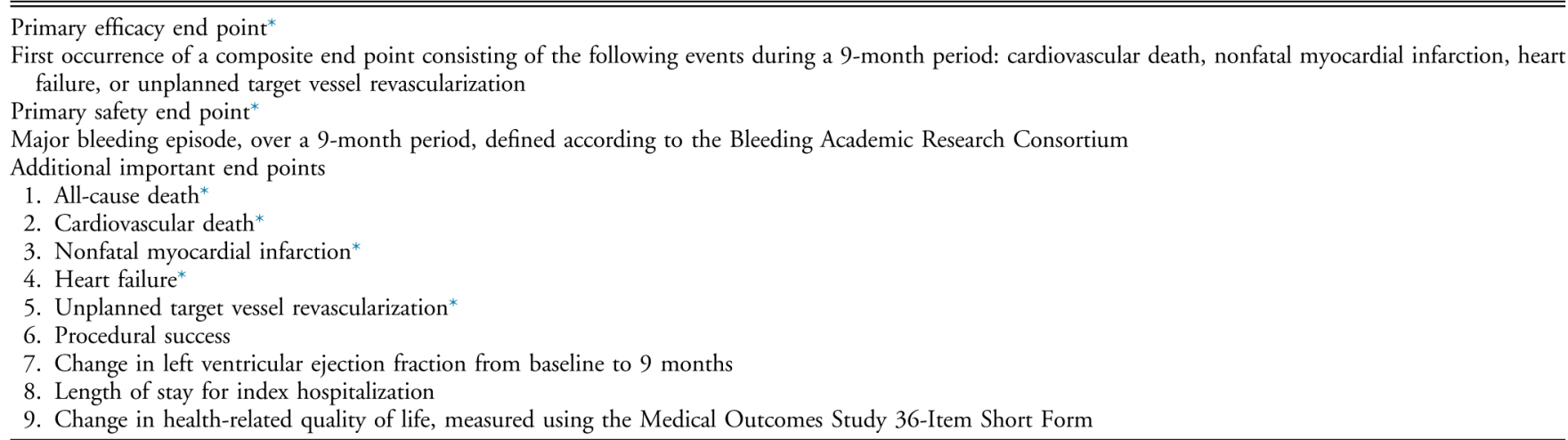

PRIMACY, Primary Reperfusion SeCondarY Stenting.

* Events adjudicated by the Clinical Event Committee.

PRIMACY participants with no events will be right-censored at 9 months. PRIMACY started with a noninformative prior, but was designed to incorporate newly available, credible external evidence as it proceeded. Because the appreciation of the nature of previous evidence is subjective (and therefore potentially controversial), a Pre and Post Probability Board (PBBB) was created to counsel the study committees and guide the incorporation of credible evidence. Although little practical guidance is available to help quantify how and to what extent previous data can be borrowed, the PBBB decided that only patient-level data from randomized controlled trials (RCTs) that investigated the delayed stenting hypothesis could be used. In addition, strength could be borrowed from previous trials through hierarchical models if it was determined that: (1) the results were similar between studies; and (2) if participants are exchangeable within each study, which implies that the twin trial populations are similar with regard to entry criteria, baseline characteristics, and end points collected (Table 4, Supplemental Table S1).

Risk ratios (RRs) of immediate vs delayed stenting will be the analysis method for the primary and all clinical end points. For each end point, we obtained the posterior distribution of the $\mathrm{RR}$ in a comparison of both groups. We updated the information in PRIMACY by treating it as exchangeable with the eligible RCTs and carried out an individual-level data random effects meta-analysis with a hierarchical prior distribution over the RR.

This analysis was conducted using a generalized linear model with a $\log \operatorname{link}^{21}$ and adjusted for the following key prerandomization covariates, for all studies: age, sex, body weight, systolic blood pressure, heart rate, diabetes mellitus, time from onset of symptoms to intervention, and creatinine. For the primary end point and all additional end points, the study protocol defined superiority of delayed stenting over immediate stenting if the posterior probability for a $R R<1$ exceeded 95\%.

The main analysis will be performed according to intention to treat principles. Worse-case scenario sensitivity analyses will be conducted for participants lost to follow-up. Additional sensitivity analyses will be performed with the treatment actually received (per protocol) to account for participants who crossed study arms. Bayesian analyses will be carried out using WinBUGS (version 1.4.3; University of Cambridge Biostatistics Unit, Cambridge, United Kingdom). Descriptive statistics (mean, SD, median, interquartile range, frequencies, and percentages) were calculated using conventional frequentist methods to compare baseline and demographic characteristics of patients.

\section{DSMB}

The DSMB oversees the safety monitoring independently from the steering committee. The responsibility of the DSMB is to protect the ethical and safety interests of participants while preserving, as much as possible, the scientific validity of the trial. Data presented to the DSMB include raw data and probability calculations to evaluate clinically relevant trends and formulate recommendations to stop the trial for safety concern. The DSMB has the capacity to prespecify their own prior at the start and during the trial. To this end, the DSMB is provided by the $\mathrm{PBBB}$ with available credible evidence from other ongoing similar investigations elsewhere in the world. Blinded interim analyses were performed using the methods proposed by Spiegelhalter et al., ${ }^{22}$ and by Fayers et al. ${ }^{23}$ The first meeting will occur after 50 participants have been followup for 9 months. The next meeting will occur when $50 \%$ of primary end points will be positively adjudicated.

\section{Baseline characteristics}

Three hundred five participants were enrolled in PRIMACY between April 2014 and September 2017. As of August 2019, anonymized individual-level data from the DEFER (NCT01717573), ${ }^{12}$ MIMI (NCT01360242), ${ }^{13}$ DANAMI-3 (NCT01435408), ${ }^{15}$ and INNOVATION (NCT02324348 $)^{14}$ trials had been shared by the investigators and included in the principal Bayesian analysis. All of these trials were reported after the randomization of the first PRIMACY participants in April 2014. For the pooled Bayesian analysis, an additional 1568 participants were included in the analysis. Baseline characteristics of the participants are presented in Table 4 for each trial. In PRIMACY, the median age at randomization was 59 years, $81 \%$ were male, and $3 \%$ had a history of myocardial infarction or PCI. All patients were hemodynamically stable. The median time from symptoms onset to intervention was 180 minutes (interquartile range, 129-301 minutes). Overall, the population of patients included in PRIMACY was comparable with those included in the other 4 trials. 


\section{Study management}

The trial is independently coordinated by the Montreal Health Innovation Coordinating Center and managed by steering committee. The trial is registered at www. ClinicalTrials.gov (NCT01542385). Members of the study committees are listed in Supplemental Appendix S2 and written informed consent of particpants is listed in Supplemental Appendix S3.

\section{Discussion}

The PRIMACY trial is designed to add substantive information to the complex question of delayed stenting in patients with STEMI. To our knowledge, PRIMACY is the first trial in cardiology to investigate a medical strategy using Bayesian inferences to inform a pretrial probability of efficacy from individual-level data reported from previous RCTs. Although other RCTs have used Bayesian inferences to study sponsored drugs or devices, none used informative prior and none addressed a medical strategy. Our alliance brings together academicians and scientists from Canada, France, Denmark, United Kingdom, and South Korea and summarizes the global experience of every single patient ever randomized into a published RCT that compared immediate with delayed stenting in patients with STEMI. The methods outlined herein offer a unique opportunity for academic investigators to study challenging, and often underfunded, medical conundrums.

In the perspective where duplicative studies are frequently conducted by independent teams, we propose that Bayesian inference can be used to combine multiple twin RCTs provided that some conditions are respected. For instance, the US Food and Drug Administration guidance for the use of Bayesian statistics in medical device clinical trials ${ }^{24}$ states that borrowing strength from other studies can be done using hierarchical models when results are similar between studies and participants are exchangeable within each study. No scale currently exists to assess intertrial exchangeability but various models recently have been proposed. For trials testing medical devices, investigators have proposed the use of robustified historical prior, which is a function that down-weights historical information if it is inconsistent with the new data. ${ }^{25}$ In PRIMACY, we relied on a pre- and post probability board to appraise and weight available evidence, to select exchangeable RCTs and we further limited between-trial heterogeneity by performing individual-level meta-regression of important prerandomization characteristics. In doing so, we opted to preserve the full weight of empirical historical patient-level information. Additionally, we used a random effects metaanalysis (compared with simple pooling) to minimize the effect of the variability on outcomes measured between studies. The open-label design will likely affect how participants are managed post randomization. For this reason, treatment imbalances (such as the intensity of heart failure medication) will have to be assessed carefully and accounted for.

In our case, all of the available studies were RCTs, addressed the same hypothesis, and had similar baseline characteristics. Open access to previous RCT data and the use of Bayesian inferences to synthesize available evidence are tools to mitigate uncertainty on the variation in association and effect. The investigation of medical strategies, as opposed to new drugs or devices, implies that heterogeneity in clinical presentations and patients' characteristics might not have been captured in the design of the trial. The Bayesian design described herein contextualizes the interpretation of study results, away from conventional, dichotomizing $P$ values, in deciding whether the observed outcomes refute our scientific hypothesis. $^{26}$

In conclusion, the final results of the PRIMACY principal hierarchical analysis are expected to show an updated posterior probability on the early hazard of reinfarction vs the expected long-term benefits on clinically important outcomes. Using the Bayesian posterior probabilities, we expect to clarify the relative merit of delaying stent implantation in patients with STEMI treated using primary PCI.

\section{Funding Sources}

The trial was supported by an operating grant from the Canadian Institute of Health Research Industry-Partnered Collaborative Research program (grant 298937), in collaboration with Boston Scientific and AstraZeneca (unrestricted grant support). Dr E. Marc Jolicoeur holds a midcareer award from les Fonds la Recherche du Québec en santé (Junior 2), and is supported by la Fondation de l'Institut de Cardiologie de Montréal.

\section{Disclosures}

The investigators disclose the following relationships: E. Marc Jolicoeur: Advisory Board: Institut National d'Excellence en Santé et Services Sociaux du Québec, Servier, Imbria Pharmaceutical, Carré technology; Board of Directors, Société de Sciences Vasculaires du Québec; DSMB: XyloCor; research grants; Boston Scientific, AstraZeneca; The University of Glasgow (employer, C. Berry) holds research and/or consultancy agreements with AstraZeneca, Abbott Vascular, Boehringer Ingelheim, GSK, HeartFlow, Novartis, and Siemens Healthcare; François Roubille: Advisory Board: Abbott, Air liquide, Research grants; Boston Scientifics, AstraZeneca; consultancy agreements with AstraZeneca, Abbott Vascular, Air liquide, Amgen, Boehringer Ingelheim, GSK, MSD, Novartis, Sanofi, Novartis, Novonordisk, and Vifor; Geraud Souteyrand: consultancy agreements: Medtronic, Abbott, and Terumo; Guillaume Cayla: consultancy agreements: Astra Zeneca, Amgen, Abbot Vascular, BMS, Boston, Biotronik, Medtronic, Pfizer, and Sanofi; Nicolas Delarche: Advisory Board: Novartis; Samer Mansour: consultant/Advisory Board/ Speaker's Bureau: Abbott Vascular, Soundbite, Boehringer Ingelheim, AstraZeneca, Bayer, BMS-Pfizer Alliance, Medtronic, Amgen, Sanofi, Servier, Gilead, and Novartis; Erick Schampaert: consultant/Advisory Board/Speaker's Bureau: Abbott, AstraZeneca, Bayer, BMS-Pfizer Alliance, Medtronic, Philips-Volcano, and Servier; Loic Belle: research grants: AstraZeneca, Medtronic, Biotronic, Boston Scientific, Abbott Vascular, and Terumo. The remaining authors have no conflicts of interest to disclose.

\section{References}

1. Reed GW, Rossi JE, Cannon CP. Acute myocardial infarction. Lancet 2017;389:197-210. 
2. Tran DT, Ohinmaa A, Thanh NX, Welsh RC, Kaul P. The healthcare cost burden of acute myocardial infarction in Alberta, Canada. Pharmacoecon Open 2018;2:433-42.

3. Jolly SS, Cairns JA, Yusuf S, et al. Randomized trial of primary PCI with or without routine manual thrombectomy. N Engl J Med 2015;372: 1389-98.

4. Stone GW, Grines CL, Cox DA, et al. Comparison of angioplasty with stenting, with or without abciximab, in acute myocardial infarction. N Engl J Med 2002;346:957-66.

5. Nordmann AJ, Bucher H, Hengstler P, Harr T, Young J. Primary stenting versus primary balloon angioplasty for treating acute myocardial infarction. Cochrane Database Syst Rev 2005;2:CD005313.

6. Wallentin L, Becker RC, Budaj A, et al. Ticagrelor versus clopidogrel in patients with acute coronary syndromes. N Engl J Med 2009;361: 1045-57.

7. Cassese S, Belle L, Ndrepepa G, et al. Deferred vs immediate stenting in primary percutaneous coronary intervention: a collaborative meta-analysis of randomized trials with cardiac magnetic resonance imaging data. Can J Cardiol 2018;34:1573-80.

8. Jolicoeur EM, Tanguay JF. From primary to secondary percutaneous coronary intervention: the emerging concept of early mechanical reperfusion with delayed facilitated stenting-when earlier may not be better. Can J Cardiol 2011;27:529-33.

9. Freixa X, Belle L, Joseph L, et al. Immediate vs. delayed stenting in acute myocardial infarction: a systematic review and meta-analysis. EuroIntervention 2013;8:1207-16.

10. Isaaz $\mathrm{K}$, Robin $\mathrm{C}$, Cerisier A, et al. A new approach of primary angioplasty for ST-elevation acute myocardial infarction based on minimalist immediate mechanical intervention. Coron Artery Dis 2006;17:261-9.

11. Hicks KA, Mahaffey KW, Mehran R, et al. 2017 Cardiovascular and stroke end point definitions for clinical trials. J Am Coll Cardiol 2018;71: 1021-34.

12. Carrick D, Oldroyd KG, McEntegart M, et al. A randomized trial of deferred stenting versus immediate stenting to prevent no- or slow-reflow in acute ST-segment elevation myocardial infarction (DEFER-STEMI). J Am Coll Cardiol 2014;63:2088-98.

13. Belle L, Motreff P, Mangin L, et al. Comparison of immediate with delayed stenting using the minimalist immediate mechanical intervention approach in acute ST-segment-elevation myocardial infarction: the MIMI study. Circ Cardiovasc Interv 2016;9:e003388.

14. Kim JS, Lee HJ, Woong Yu C, et al. INNOVATION study (Impact of Immediate Stent Implantation Versus Deferred Stent Implantation on Infarct Size and Microvascular Perfusion in Patients With
ST-Segment-Elevation Myocardial Infarction). Circ Cardiovasc Interv 2016;9:e004101.

15. Kelbaek H, Hofsten DE, Kober L, et al. Deferred versus conventional stent implantation in patients with ST-segment elevation myocardial infarction (DANAMI 3-DEFER): an open-label, randomised controlled trial. Lancet 2016;387:2199-206.

16. Berry DA. Bayesian clinical trials. Nat Rev Drug Discov 2006;5:27-36.

17. Thygesen K, Alpert JS, Jaffe AS, et al. Third universal definition of myocardial infarction. J Am Coll Cardiol 2012;60:1581-98.

18. Mehran R, Rao SV, Bhatt DL, et al. Standardized bleeding definitions for cardiovascular clinical trials: a consensus report from the Bleeding Academic Research Consortium. Circulation 2011;123:2736-47.

19. Joseph L, du Berger R, Belisle P. Bayesian and mixed Bayesian/likelihood criteria for sample size determination. StatMed 1997;16:769-81.

20. Pfeffer MA, Braunwald E, Moye LA, et al. Effect of captopril on mortality and morbidity in patients with left ventricular dysfunction after myocardial infarction. Results of the survival and ventricular enlargement trial. The SAVE Investigators. N Engl J Med 1992;327:669-77.

21. Torman VB, Camey SA. Bayesian models as a unified approach to estimate relative risk (or prevalence ratio) in binary and polytomous outcomes. Emerg Themes Epidemiol 2015;12:8.

22. Spiegelhalter DJ, Freedman LS, Parmar MK. Bayesian approaches to randomized trials. J R Stat Soc A 1994;157:357-416.

23. Fayers PM, Ashby D, Parmar MK. Tutorial in biostatistics Bayesian data monitoring in clinical trials. Stat Med 1997;16:1413-30.

24. US Food and Drug Administration: Guidance for the Use of Bayesian Statistics in Medical Device Clinical Trials. Available at: http://www.fda. gov/MedicalDevices/DeviceRegulationandGuidance/GuidanceDocuments/ ucm071072.htm. Accessed January 20, 2020.

25. Schmidli H, Gsteiger S, Roychoudhury S, et al. Robust meta-analyticpredictive priors in clinical trials with historical control information. Biometrics 2014;70:1023-32.

26. Amrhein V, Greenland S, McShane B. Scientists rise up against statistical significance. Nature 2019;567:305-7. 